\title{
Face Analysis Using Row and Correlation Based Local Directional Pattern
}

\author{
Srinivasa Perumal $\mathrm{R}^{*}$ and Chandra Mouli P.V.S.S.R ${ }^{+}$ \\ * School of Information Technology and Engineering, Vellore Institute of Technology, Vellore, Tamil Nadu, India \\ + Department of Computer Science, Central University of Tamil Nadu, Thiruvarur, Tamil Nadu, India
}

Received 7 July 2020; Accepted 19 September 2020

\begin{abstract}
Face analysis, which includes face recognition and facial expression recognition, has been attempted by many researchers and proposed ideal solutions. The problem is still active and challenging due to an increase in the complexity of the problem viz. due to poor lighting, face occlusion, low-resolution images, etc. Local pattern descriptor methods has been introduced to overcome these critical issues to improve the recognition rate. These methods extract the discriminant information from the local features of the face image for recognition. In this paper, local descriptor based two methods, namely row-based local directional pattern and correlation-based local directional pattern are proposed by extending an existing descriptor local directional pattern (LDP). Further, the two feature vectors obtained by these methods are concatenated to form a hybrid descriptor. Experimentation has been carried out on benchmark databases and results infer that the proposed hybrid descriptor outperforms the other descriptors in face analysis.
\end{abstract}

Key Words: Local Directional Pattern, Face Recognition, Image Descriptor, Feature Descriptor, Face Descriptor, Face Detection.

\section{Introduction}

Face recognition has become an active research area for the past two decades.Defining a robust descriptor for different pose variations, illumination changes, expression changes and images captured after a certain duration of time are some of the most challenging tasks. Statistical feature extraction has been rigorously used for addressing these challenges. A statistical local descriptor extracts unique features of the face to identify a person. Local pattern feature extraction method addresses these challenges. The local pattern descriptor based methods gained the attention of many researchers and identified the relativity of the neighbour and reference pixel to extract the structure of an image [29].

Ojala et al. [1] proposed a local binary pattern (LBP) descriptor for describing the texture information and is widely used in different applications. Ahonen et al. [8] proposed LBP to define the shape of the facial component. In the LBP mechanism, an image divided into small blocks of size $p \times p$. For each $p \times p$ block, the center pixel value compared with its eight neighbours. If the value of the center pixel is less than the neighbour

Correspondence to: <chandramouli@cutn.ac.in>

Recommended for acceptance by $<$ Angel D. Sappa $>$

https://doi.org/10.5565/rev/elcvia.1254

ELCVIA ISSN:1577-5097

Published by Computer Vision Center / Universitat Autònoma de Barcelona, Barcelona, Spain 
pixel value, then the neighbour pixel position is set to 1 ; otherwise, it is set to 0 . For each pixel value, a new eight-bit code is assigned based on the center pixel and its neighbours. LBP encoded value is computed using Equation (1). The resultant LBP encoded image further divided into $8 \times 8$ non-overlapping sub-regions. For each $8 \times 8$ region, a histogram is generated and the corresponding bins are used to represent the feature vector. The feature vector, thus formed for every region, is concatenated to form a single feature vector to represent the face. LBP is an efficient method and eases the feature extraction compared to other methods. LBP performance varies if the image contains random noise or pose variations or illumination variations or expression variations. Ojala et al. extended the LBP operator to recognize the uniform pattern [7]. This method is robust to scale variations. The primary uniform patterns extracted by LBP include corners, edges, and local patches because they represent the discriminant information of the object. LBP is simple and computationally efficient.

$$
L B P_{P, R}\left(x_{c}, y_{c}\right)=\sum_{p=0}^{P-1} s\left(g_{p}-g_{c}\right) \times 2^{p} ; \quad s(x)= \begin{cases}1, & x>=0 \\ 0, & \text { otherwise }\end{cases}
$$

where $P$ represents the number of sampling points on a circle of radius $R$. Regularly used LBP is $L B P_{8,2}$.

Ahonen et al. applied LBP for face recognition [10]. Weighted Chi-square distance metric is employed as a dissimilarity measure between two face images. In feature-based methods, LBP has changed the dimensions of research in face recognition into a different arena. Another variation of LBP is called uniform LBP. A uniform LBP is an LBP pattern with only two transitions from 0 to 1 or vice-versa. Local Phase Quantization (LPQ) is a descriptor for texture description [12]. This descriptor performs better when compared to LBP and robust in case of blurred images. Local Ternary Pattern (LTP) [17] is a similar descriptor that extracts discriminant information beter than LBP and is resistant to noise. LTP is an eight-bit code with ternary values $\{-1,0$, or 1$\}$.

Zhang et al. proposed a local derivative pattern $\left(L D_{e} P\right)$ to overcome the pitfalls of LBP [18]. $L D_{e} P$ has explored the direction information of the neighbourhood instead of intensity values. Jabid et al. have introduced a local directional pattern(LDP) for face recognition [14]. They used LDP for gender classification, facial expression identification, and object recognition $[15,16]$. LDP is robust to noise and poor lighting conditions. Local directional number pattern (LDNP) is another variation of LDP proposed by Ramirez et al. [22] to overcome the above problem. LDNP is a six-bit binary code assigned to each pixel which represents both spatial and intensity transitions. The code can be generated by analyzing the edge responses from convoluting Kirsch masks $\left(M_{0}, M_{1}, \cdots, M_{7}\right)$ with face image. The topmost positive pixel position generates a three-bit code and the minimum negative pixel position generates a three-bit code; together forms the six-bit code in LDNP. The authors have further tried the LDNP approach by using Gaussian mask instead of Kirsch masks. The LDNP pattern with a Gaussian mask labelled as Local Directional Number pattern with the Gaussian function (LDNG). LDNG has improved the accuracy of face recognition than LDNP.

Kabir et al. proposed a similar descriptor called local directional pattern variance (LDPv), which characterizes the local information as well as the spatial structure of LDP [20]. The dimension of the feature vector is reduced using PCA and support vector machines (SVM) is used for classification. LDPv extracts the useful range of discriminant features for a low-resolution image. Enhanced local directional pattern (EnLDP) [23] follows the same procedure of LDP to extract the edge response values, but EnLDP generates an octal code instead of binary code. EnLDP selects the top two prominent directions to create a code. Ishraque et al. proposed a descriptor for facial expression recognition and labelled as local Gabor directional pattern (LGDP) [21]. LGDP used Gabor wavelet as a filter to encode the local structure information of the face image, unlike other stateof-the-art methods that used Kirsch masks. LGDP follows the same steps as LDP for generating histogram and face recognition. Liu et al. extended the descriptor for face recognition with the dominant direction to improve the recognition rate and reduce the computational complexity [26]. Gabor feature outperformed the other methods for face recognition, but it has some disadvantages. The major drawback of the Gabor feature is that the length of the feature descriptor is higher when compared to other methods. Gabor feature requires more processing time and memory space to store the feature vector due to high dimensional feature space. Fathi et al. [28] introduced rotation invariant multi-scale descriptor using Gabor filters. This feature descriptor, along 
with local features, achieved better recognition rate on face recognition.

Nguyen and Caplier developed a global feature vector by concatenating BELBP and LPQ descriptors [27]. Local patterns of gradients (LPOG) used block-wise elliptical LBP (BELBP), Whitened Principal Component Analysis (WPCA) for dimension reduction and weighted angle based distance for classification. LPOG is faster than other local pattern descriptors. The high dimensional feature space improves the discriminative information for face recognition [32]. To reduce the complexity, the local descriptors are incorporated for dimension reduction. Perumal and Mouli proposed the dimensionality-reduced local directional pattern for face recognition [31]. It reduced the feature vector length and improved the results on standard benchmark databases. The authors extended their method to the second level for face recognition [30]. Further, they modified their method for face recognition and facial expressions to improve the recognition rate [33, 34].

In this paper, two methods namely, row-wise LDP and correlation based LDP are proposed. The corresponding code patterns obtained are combined to form a single eight-bit code for a pixel. The features are extracted from each region and concatenated to create a feature vector.

The remaining sections of the paper are organized as follows. The proposed RC-LDP discussed in section 2 and the face description is elaborated in section 3. Experimental results and performance analysis carried out is analyzed in section 4 and conclusions of the paper are presented in Section 5.

\section{Row and Correlation based Local Directional Pattern}

A row and correlation based local directional pattern (RC-LDP) is an extended version of LDP. The given image is divided into $3 \times 3$ overlapping regions and each region is convoluted with eight directional Kirsch masks, from which eight responses are obtained. Encoding has been done in two ways. Row-based encoding considers the row-wise Kirsch mask responses. Correlation-based encoding considers two adjacent triangles formed from the center pixel $x_{c}$ with the two adjacent responses. Instead of choosing top $k$ absolute values from the edge responses, RC-LDP encodes the image in two ways to select the prominent directions from the edge responses. In LDP, the eight directions are not treated equally and hence some valuable information might be lost. RC-LDP considers both the negative and positive sign directions to select the significant directions of the regions. The block diagram of the proposed method shown in Figure 1.

\subsection{Row Based Local Directional Pattern}

In LDP, the top three responses are chosen and their positions set to 1 . This method ensures that there exist three 1's in every binary pattern of the LDP encoded value. In row-based local directional pattern also, three 1 's exist in every binary pattern of the encoded value, but the 1's are chosen in a way that a maximum response from every row is considered and the corresponding position is set to 1 . The prominent direction of the neighbourhood is calculated with Kirsch masks in eight directions. The directional number defined as

$$
P_{\text {dir }}^{n}=\operatorname{argmax}_{i}\left\{\Pi_{i} \mid 0 \leq i \leq 7\right\}
$$

where $P$ represents the prominent direction of the neighbourhood.

$$
\Pi_{i}=\left|I_{x} * m_{i}\right|
$$

where $I_{x}$ is the absolute response of the convolution of a sub-region and $m_{i}$ is $i^{t h}$ Kirsch mask.

Let $\left\{M_{0}, M_{1}, \cdots, M_{7}\right\}$ be the eight responses obtained by convoluting a $3 \times 3$ region with the eight directional Kirsch masks. The row based local directional pattern selects the maximum responses from each row $P^{1}, P^{2}, P^{3}$. Equations (4), (5), and (6) define the three maximum responses from the three rows of the $3 \times 3$ regions. The significant direction consists of structure information of the sub-region.

$$
P^{1}=\max \left\{M_{1}, M_{2}, M_{3}\right\}
$$




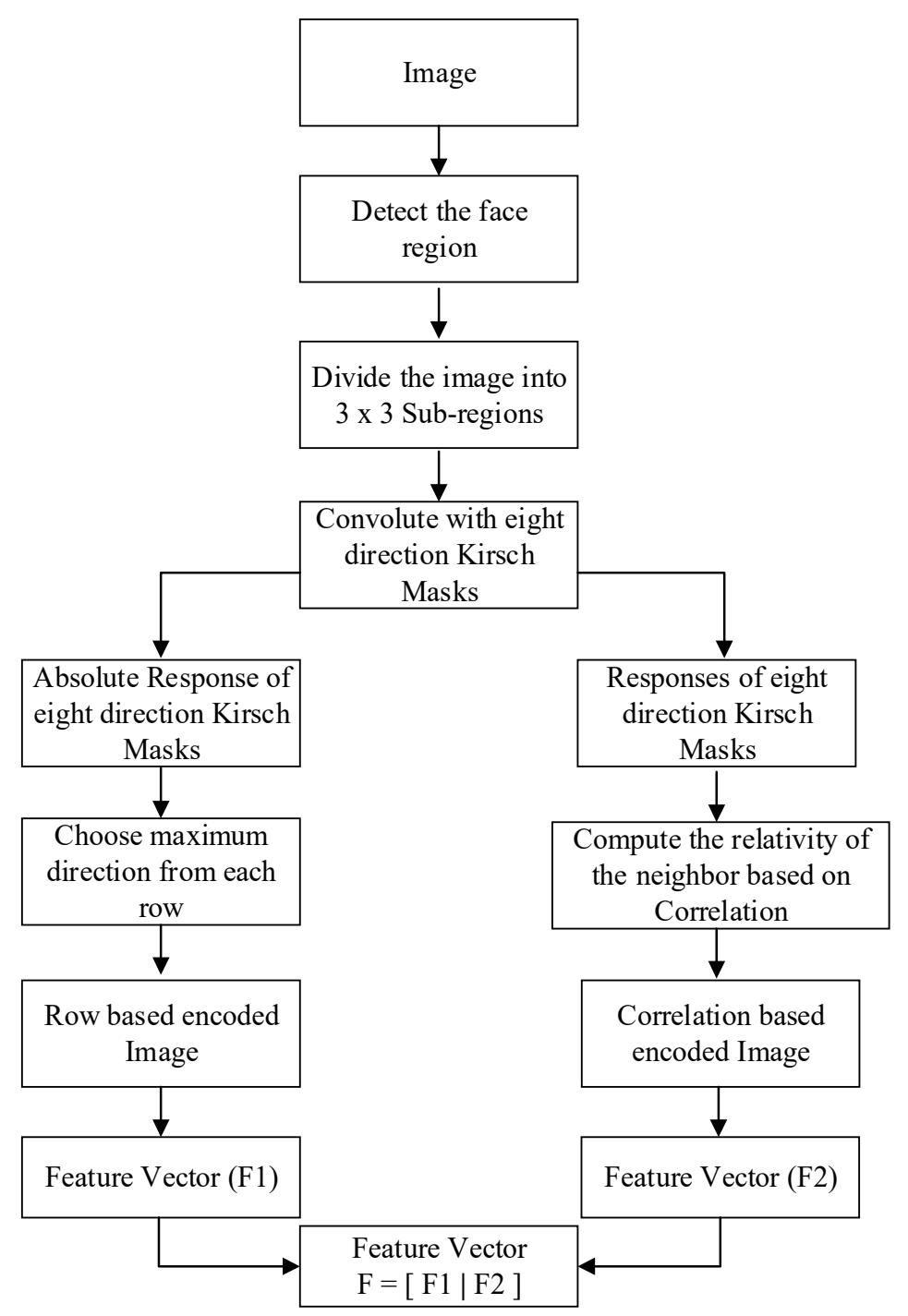

Figure 1: Block Diagram of RC-LDP

$$
\begin{gathered}
P^{2}=\max \left\{M_{0}, M_{4}\right\} \\
P^{3}=\max \left\{M_{5}, M_{6}, M_{7}\right\}
\end{gathered}
$$

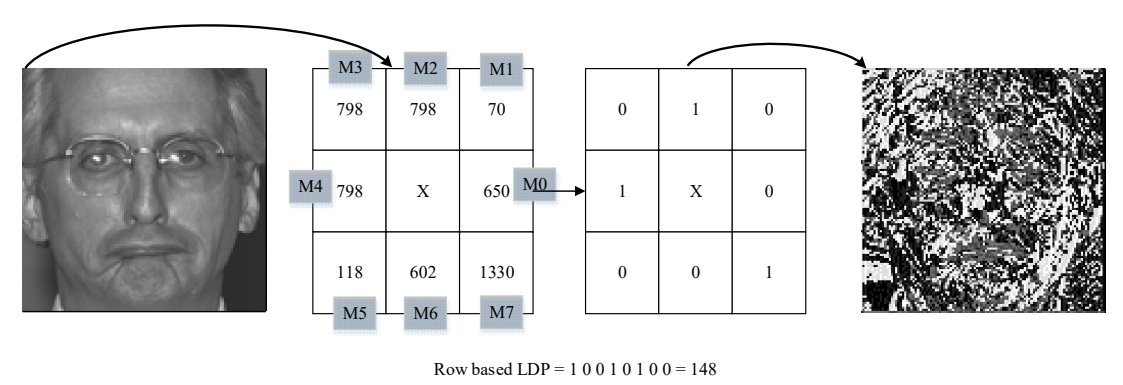

Figure 2: Illustration of Row based LDP 
The pattern is formed by selecting the $P^{1}, P^{2}, P^{3}$. The direction chosen position is set to 1 and the rest are set to 0. For example, consider the neighbourhood shown in Figure 2. First, determine the eight directional responses by Kirsch masks. Then $P^{1}$ is selected from the responses of $M_{1}, M_{2}, M_{3}, P^{2}$ is selected from responses of $M_{0}, M_{4}$ and $P^{3}$ is chosen from $M_{5}, M_{6}, M_{7}$. Finally, the code is generated for the center pixel.

\subsection{Correlation Based Local Directional Pattern}

Correlation based local directional pattern identifies the relationship with adjacent neighbouring pixels. Each center pixel has adjacent neighbouring pixels in eight directions. Eight neighbourhood is used to form a pattern for the center pixel. The image was divided into $3 \times 3$ sub-regions. In each region, the correlation between a set of three responses considered - the three responses considered such that two adjacent triangular shapes are formed with center element. If the correlation between the two pairs exceeds the threshold value, then the center of the corresponding three pixels is set to 1 . The formation of the pattern described in Figure 3. The relative pattern calculated is defined in Equations (7) to (14).

$$
\begin{aligned}
& R_{1}=\text { Correlation }\left(\left\{X_{c}, M_{0}, M_{1}\right\},\left\{X_{c}, M_{0}, M_{7}\right\}\right) \\
& R_{2}=\text { Correlation }\left(\left\{X_{c}, M_{1}, M_{2}\right\},\left\{X_{c}, M_{1}, M_{0}\right\}\right) \\
& R_{3}=\text { Correlation }\left(\left\{X_{c}, M_{2}, M_{3}\right\},\left\{X_{c}, M_{2}, M_{1}\right\}\right) \\
& R_{4}=\text { Correlation }\left(\left\{X_{c}, M_{3}, M_{4}\right\},\left\{X_{c}, M_{3}, M_{2}\right\}\right) \\
& R_{5}=\text { Correlation }\left(\left\{X_{c}, M_{4}, M_{5}\right\},\left\{X_{c}, M_{4}, M_{3}\right\}\right) \\
& R_{6}=\text { Correlation }\left(\left\{X_{c}, M_{5}, M_{6}\right\},\left\{X_{c}, M_{5}, M_{4}\right\}\right) \\
& R_{7}=\text { Correlation }\left(\left\{X_{c}, M_{6}, M_{7}\right\},\left\{X_{c}, M_{6}, M_{5}\right\}\right) \\
& R_{8}=\text { Correlation }\left(\left\{X_{c}, M_{7}, M_{0}\right\},\left\{X_{c}, M_{7}, M_{6}\right\}\right)
\end{aligned}
$$

where $M_{0}, M_{1}, \cdots, M_{7}$ are the eight responses obtained by convoluting a $3 \times 3$ region with the eight directional Kirsch masks and $X_{c}$ denotes center position value of the region.

The resultant pattern may have all 1's or all 0's. The constraint in the LDP code is slightly relaxed in the correlation based LDP. It is proposed that the resultant code may have at most three 1's, which allows it to accept the code pattern containing zero to three 1's. If the number of 1's in the resultant code pattern exceeds three, the 1's in the most significant bits are retained, and the rest are set to 0 . This method results in a total of 93 distinct patterns.

Correlation extracts the most prominent feature because it defines the relationship between the neighbours. However, the linear correlation is used to determine the relationship between the center pixel and the adjacent neighbourhood-the correlation defined in Equation (15). The row based local directional pattern extracts local information and the relative pattern is also helpful in forming a better information feature vector.

$$
r=\frac{\Sigma(X-\bar{X})(Y-\bar{Y})}{\sqrt{\Sigma(X-\bar{X})^{2}} \sqrt{\Sigma(Y-\bar{Y})^{2}}}
$$




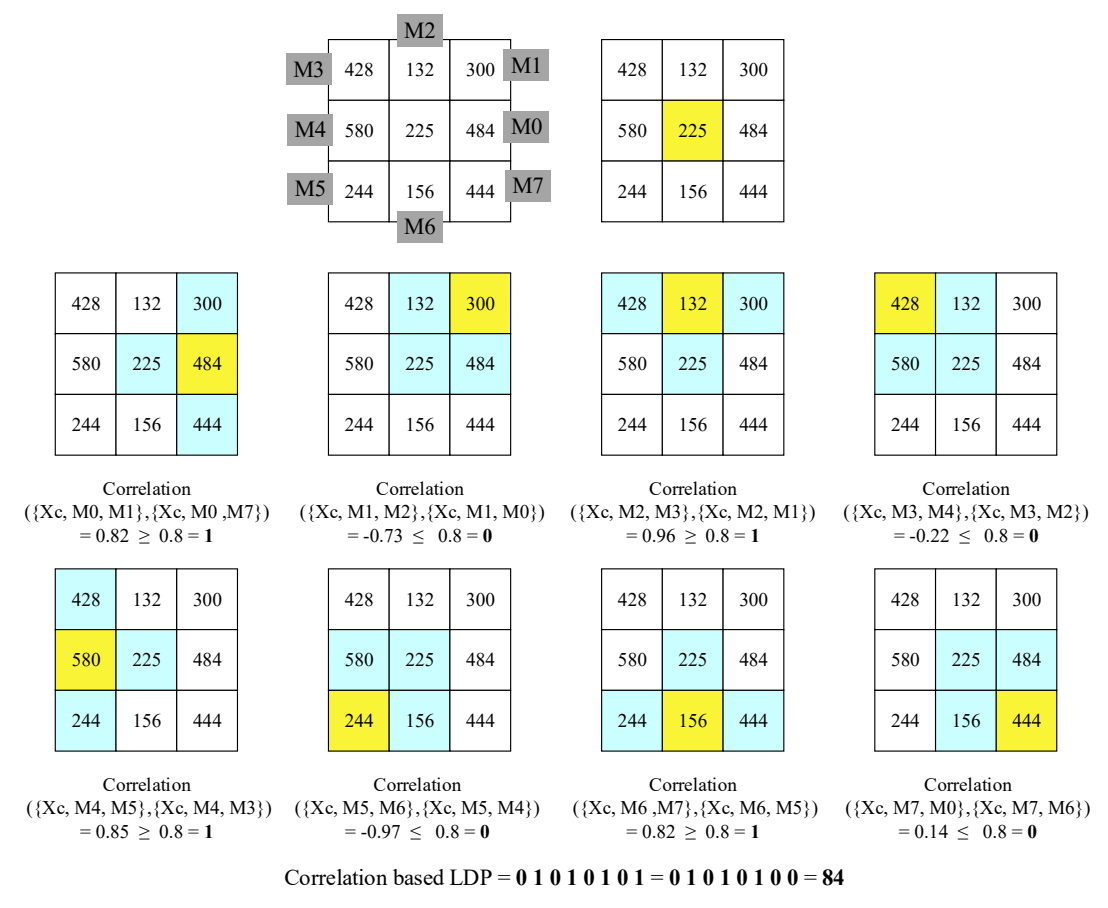

Figure 3: Correlation based local directional pattern

\section{Face Description}

An RC-LDP histogram represents each face. Every pixel of RC-LDP extracts the edges, corners and other textural information. The row based encoded image divided into $m \times m$ sub-regions. Histogram describes the distribution of the local patterns from the image. Here the histogram extracts 93 patterns from each region out of 256 patterns. Similarly, the correlation based encoded image divided into $m \times m$ regions and a histogram descriptor extracted-the histogram bins of both the encoded images concatenated to form a global descriptor of the given image. Figure 4 shows the face description process of RC-LDP. The histograms are accumulated using Equations (16) and (17)respectively.

$$
H_{n}(c)=\sum_{x, y} f\left(R_{i}(x, y)=C_{i}\right), C_{i}=i^{t h} L D P \text { Pattern }
$$

where $R_{i}$ is the $8 \times 8$ region of Row based encode image / Correlation based encoded image.

$$
H_{n}(c)=\sum_{\substack{x, y \in R_{n} \\ R L D P(x, y) \mid C L D P(x, y)=c}} \nu, \forall c
$$

where $c$ is RC-LDP code and $\nu$ is the number of bins in a region.

\subsection{Face Recognition and Facial Expression Recognition}

The feature vector for every image is stored in the database. For a given query image, a feature vector is computed. The Chi-square dissimilarity measure is used to compare the query image feature descriptor with the feature descriptors in the database. The lowest measure value for the selected candidate indicates that a match has found. The Chi-square dissimilarity measure for two feature vectors of length $L$ defined in Equation (18). 


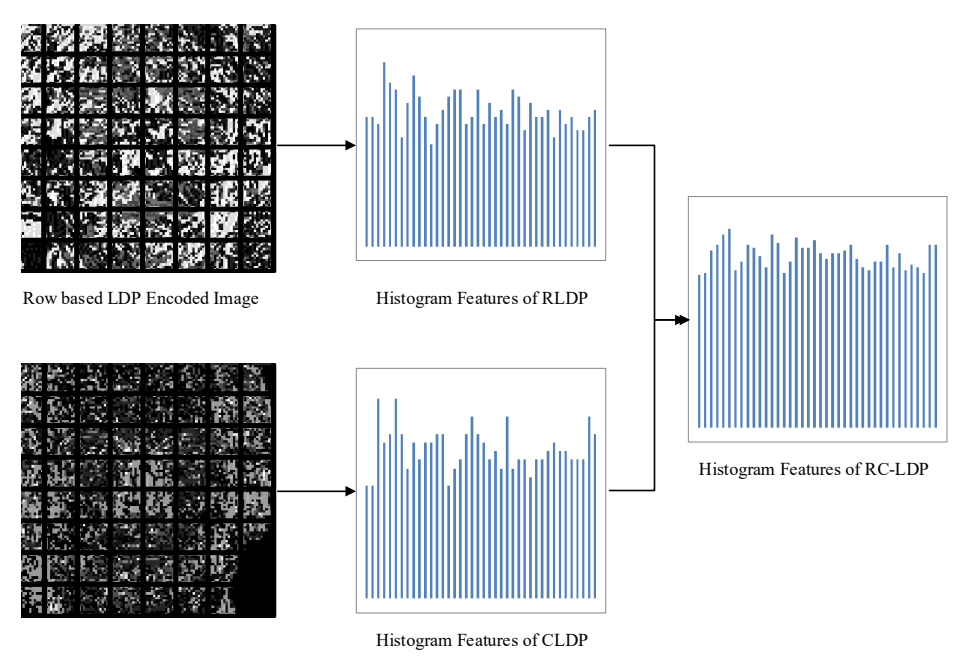

Figure 4: Face Description by Histogram

$$
\chi^{2}\left(f_{1}, f_{2}\right)=\sum_{i=1}^{M} w_{i} \frac{\left(f_{1}(i)-f_{2}(i)\right)^{2}}{f_{1}(i)+f_{2}(i)}
$$

where $w_{i}$ indicates the weight of the $i^{\text {th }}$ region

The 93 patterns generated in RC-LDP fall within the range of $[0-255]$, which are divided into four equal parts in multiples of 64 . A weight of 0.25 is assigned to first part [0 - 63]. Similarly, weights $0.5,0.75$, and 1 assigned to the other three parts, respectively. For a region $i$, the weight is assigned as follows: Check the maximum number of patterns that occur in that region; the weight is determined based on to which part the maximum occurring pattern belongs to. From physiological findings, the small portion of the face like eyes, nose and mouth has discriminant features of the person. The proposed method assigns a weight for each subregion based on the occurrences of the information.

The performance of the facial expression recognition can be evaluated using SVM. SVM is a powerful technique for classification. SVM implicitly maps the data into a higher dimensional feature space.

Given a training set of labelled examples $T=\left(x_{i}, y_{i}\right), i=1, \ldots, M$ where $x_{i} \in R^{n}$ and $y_{i} \in[-1,1]$, the new test data will be classified by Equation (19).

$$
f(x)=\operatorname{sign}\left(\sum_{i=1}^{M} \alpha_{i} y_{i} K\left(x_{i}, x\right)+b\right)
$$

where $\alpha_{i}$ are Lagrange multipliers, $\mathrm{b}$ is a bias and $\mathrm{K}$ is a kernel function.

The training images $x_{i}$ with $\alpha_{i}>0$ are support vectors, and the separating hyperplane maximizes the margin concerning support vectors. The method is analysed with various kernels like polynomial, linear and radial basis function kernels. To determine the performance of each subject, 10-fold cross-validation testing scheme implemented.

\section{Experimental Results and Analysis}

The proposed method has experimented on face recognition and facial expression recognition database. The performance of the method is calculated based on the confusion matrix. Confusion matrix is a performance measurement for classification problem where output can be 2 or more classes. It is used in model evaluation, monitoring and managing the model. The method reduces the effects of expression changes, occlusion, and 
illumination changes and extracts a very informative feature for representing the face. The proposed method is tested on two types of standard benchmark databases - for face recognition and facial expression recognition. The standard databases used for face recognition include FERET [5], YALE [9], ORL [6], AR database [2] and LFW [11]. The facial expression is defined based on the human emotional expressions such as anger, disgust, fear, happy, sad and surprise [13]. These six emotions constitute a six-class facial expression. The standard benchmark databases used for facial expression recognition include CK [4], JAFFE [3] and EMOTIW 2015 database [24].

\subsection{FERET Database}

FERET [5] database contains 14,501 images of 1010 individuals. The images are captured under various poses, illumination changes, facial expressions and ageing effects. The database is classified into five sets, such as $F a, F b, F c, d u p-I$ and $d u p-I I$. Fa set is used as a gallery image set and the rest of the image sets used as probe images.

- $F a$ consists of 1010 frontal images.

- $F b$ consists of 1009 images with different expression variations.

- Fc consists of 194 images with illumination variations.

- dup-I has 722 images captured after a certain period.

- dup-II has 234 images and a subset of dup-I.

The performance of the proposed method on 4 probe sets of the FERET database shown in Figure 5. From Figure 5, the RC-LDP outperformed the other state-of-the-art methods. This method is effective in expression changes and illumination variations because it achieved $100 \%$ and $99.97 \%$ accuracy in $F c$ and $F b$, respectively. The proposed method also achieved impressive results on ageing variation probe sets. dup-I and dup-II attained $99.5 \%$ and $97.5 \%$, which are better recognition rate when compared with other local existing descriptors.

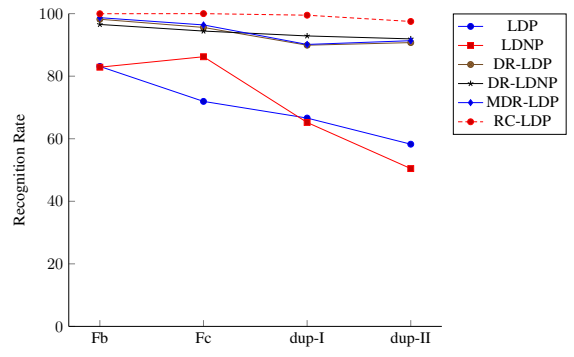

(a) Recognition Rate

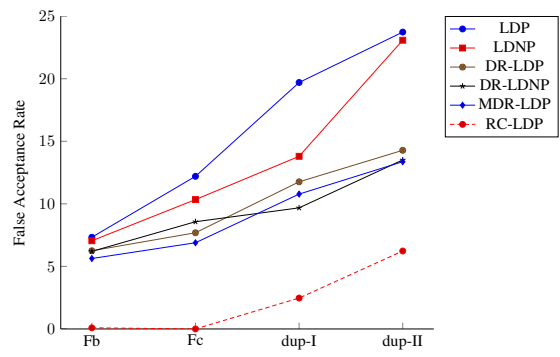

(c) False Acceptance Rate

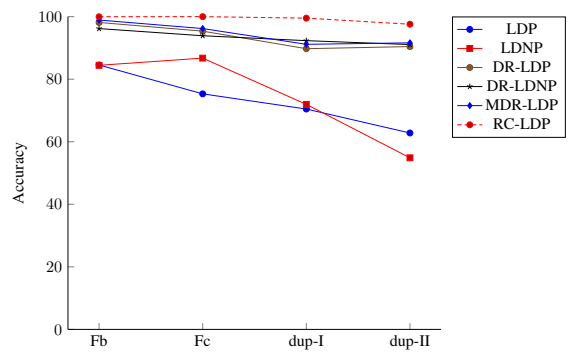

(b) Accuracy

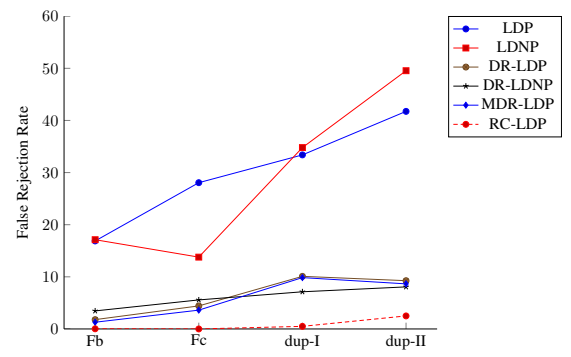

(d) False Rejection Rate

Figure 5: Performance Analysis of RC-LDP with existing methods on FERET database 


\subsection{EXTENDED YALE-B Database}

Extended Yale B database [9] consists of images with illumination variations. The database contains 16, 128 images of 28 subjects. Each subject has 9 poses in 64 different illumination conditions. It is categorized into 5 sets based on the angle among the central camera axis and light source direction, such as Sub1, Sub2, Sub3, Sub4 and Sub5. Subl used as a gallery image set and the rest of the image sets used as probe images. The subsets are categorized .

- The angle less than or equal to $12^{0}$ labelled as Subl.

- The angle between $13^{0}$ to $25^{0}$ labelled as $S u b 2$.

- The angle between $26^{0}$ to $50^{0}$ labelled as Sub3.

- The angle between $51^{0}$ to $77^{0}$ labelled as Sub4.

- The angle is higher than $77^{0}$ labelled as Sub5.

The performance of the proposed method on 4 probe sets of Extended YALE-B database is analyzed and proved that the proposed method is robust to various illumination conditions. Figure 6 shows the result of the proposed method with other state-of-the-art methods. From the results, it observed that RC-LDP is reliable in various illumination conditions. The correlation based descriptor extracts the sign direction and relativity of the neighbourhood. Due to that, the performance for an angle higher than $51^{0}$ also achieved better results in very severe illumination conditions.

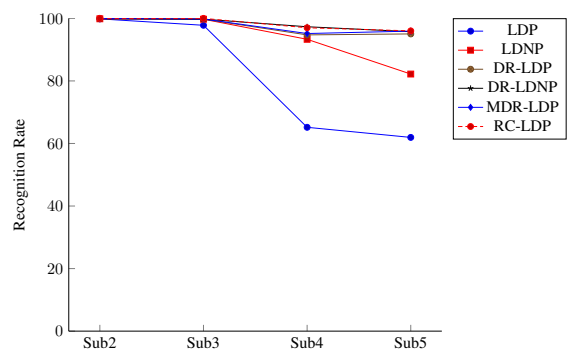

(a) Recognition Rate

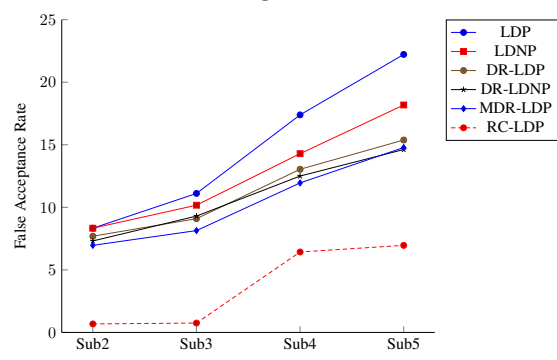

(c) False Acceptance Rate

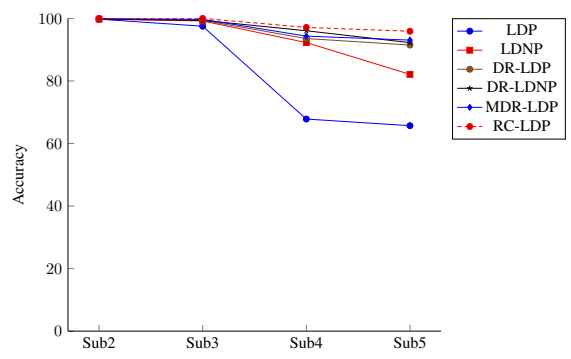

(b) Accuracy

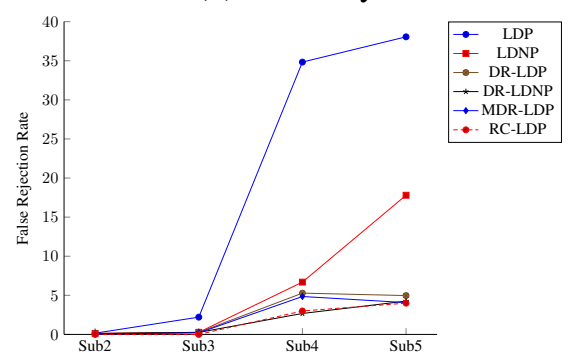

(d) False Rejection Rate

Figure 6: Performance Analysis of RC-LDP with existing methods on Extended YALE-B database

\subsection{ORL Database}

ORL database [6] consists of 400 images of 40 subjects captured under different lighting conditions, different periods and various facial expressions. Each subject contains ten images out of which two randomly selected for training and the rest for the testing set. In the ORL database 80 images used as gallery images and the remaining 320 images used as probe images. 
ORL database used to test and evaluate the performance between the RC-LDP and the state-of-the-art methods under pose variation, illumination and expression changes. The recognition rate and other metrics are shown in Table 1. The proposed method outperformed other methods among all the results. The method is robust to pose variations, expression changes and illumination variations.

Table 1: Performance Analysis of RC-LDP with existing methods on ORL database

\begin{tabular}{|c|c|c|c|c|}
\hline Method & Recognition Rate & Accuracy & False Acceptance Rate & False Rejection Rate \\
\hline LDP & 88.50 & 87.90 & 22.22 & 11.50 \\
\hline LDNP & 89.15 & 89.61 & 19.05 & 10.85 \\
\hline DR-LDP & 97.62 & 97.22 & 6.67 & 2.78 \\
\hline DR-LDNP & 97.81 & 97.45 & 6.00 & 2.19 \\
\hline MDR-LDP & 97.93 & 98.15 & 6.08 & 1.85 \\
\hline RC-LDP & $\mathbf{9 9 . 9 7}$ & $\mathbf{9 9 . 9 5}$ & $\mathbf{0 . 8 4}$ & $\mathbf{0 . 0 3}$ \\
\hline
\end{tabular}

\subsection{AR Database}

AR database [2] consists of 4000 images of 126 subjects. The database contains different facial expression images, occluded images and different illumination conditions. It consists of 50 male and 50 female subjects. Each image is captured under 4 different facial expressions, 3 illumination conditions, 2 disguise cases in the first session. In the second session, the imagess are captured under 4 illumination conditions and 4 disguise cases. The frontal images and neutral images are called gallery images and the rest of the images in the database are called test images.

In the AR database, the images are captured under different facial expressions, different illumination conditions and disguise cases. The face images are detected by Viola-Jones algorithm. The results of the AR database are shown in Table 2. For the illumination variation, expression changes and partial occlusion, the proposed RC-LDP obtained maximum recognition rate than other state-of-the-art methods.

Table 2: Recognition rate of RC-LDP with other local descriptors on AR database

\begin{tabular}{|l|c|c|c|c|}
\hline Method & Illumination & Expression & Occlusion & Mean \\
\hline LDP & 95.15 & 80.46 & 81.33 & 85.65 \\
\hline DR-LDP & 95.20 & 85.23 & 86.26 & 88.90 \\
\hline DR-LDNP & 95.73 & 86.92 & 87.15 & 89.93 \\
\hline MDR-LDP & 96.13 & 88.25 & 87.93 & 90.77 \\
\hline RC-LDP & $\mathbf{9 8 . 5 2}$ & $\mathbf{9 1 . 4 6}$ & $\mathbf{9 0 . 1 3}$ & $\mathbf{9 3 . 3 7}$ \\
\hline
\end{tabular}

\subsection{Face Verification : LFW Database}

Labeled Faces in the Wild (LFW) [11] is the most challenging database because the images are collected from the web. This database is used to understand the problem of face recognition in an unconstrained environment. LFW consists of 13, 233 images from 5, 749 subjects. The database consists of facial images with different poses, different facial expressions and occlusions [19].

In LFW-a, there are about 1056 images of 433 subjects. The proposed method randomly considers the images from the set of individuals that are distinct from one another in terms of pose, expression, background color, lighting, frontal view, etc. The proposed method test on the View 2 set of LFW, which consists of 10 folds of 300 positive and 300 negative image pairs randomly selected from the database. The mean accuracy rate of the existing method compared with the proposed method and shown in Table 3. The proposed method used the 
LFW database for face verification. The proposed method achieved better verification rate than existing local descriptors.

Table 3: Mean Accuracy Rate of RC-LDP with other local descriptors on LFW database

\begin{tabular}{|l|c|}
\hline Method & Mean Accuracy \\
\hline LDP & $71.32 \pm 0.3$ \\
\hline LDNP & $65.17 \pm 0.4$ \\
\hline DR-LDP & $73.25 \pm 0.6$ \\
\hline DR-LDNP & $73.73 \pm 0.3$ \\
\hline MDR-LDNP & $73.87 \pm 0.5$ \\
\hline RC-LDP & $\mathbf{7 8 . 2 5} \pm \mathbf{0 . 3}$ \\
\hline
\end{tabular}

\subsection{Cohn-Kanade Expression Database}

The Cohn-Kanade(CK) database [4] contains 100 subjects. The subject in the database is at the age of 18 to 30 . Of these 100 subjects, $15 \%$ of the subjects were African-American and $3 \%$ were Asian or Latino. Each subject was in different size like $640 \times 480$ or $640 \times 490$. Each subject consists of 6 emotions of the person with 23 facial expressions. For six-class expression, 408 image sequences are selected that results in 1224 expression images. For seven-class expression, 408 image sequences are selected that results in 1632 expression images. The six-class expressions are angry, disgust, happy, sad, surprise and fear. The seven-class expressions include neutral expression in addition to the other six-class expressions.

The recognition rate of six-class and seven-class expressions are shown in Tables 4 and 5. From the results, the RBF kernel outprformed than other kernels in both six-class and seven-class expression. The seven-class expression attained lower results compared with results obtained for six-class expression because the neutral expression confused with the other expressions in seven-class expression. The proposed method obtained better recognition rate on CK database. To give a clear picture, the confusion matrix is presented in Tables 6 and 7 for six-class and seven-class expressions, respectively.

Table 4: Recognition rate of six-class expression of RC-LDP using SVM on CK database

\begin{tabular}{|l|c|l|c|}
\hline \multicolumn{1}{|c|}{ Method } & Linear & Polynomial & RBF \\
\hline LDP & $94.9 \pm 1.2$ & $94.9 \pm 1.2$ & $98.5 \pm 1.4$ \\
\hline LDNP & $98.4 \pm 1.4$ & $99.1 \pm 0.7$ & $99.2 \pm 0.8$ \\
\hline DR-LDP & $96.4 \pm 1.3$ & $97.2 \pm 1.3$ & $98.7 \pm 0.9$ \\
\hline MDR-LDP & $98.8 \pm 1.2$ & $99.3 \pm 1.2$ & $99.5 \pm 0.5$ \\
\hline RC-LDP & $99.4 \pm 0.8$ & $99.5 \pm 0.8$ & $99.8 \pm 0.3$ \\
\hline
\end{tabular}

Table 5: Recognition rate of seven-class expression of RC-LDP using SVM on CK database

\begin{tabular}{|l|c|l|c|}
\hline \multicolumn{1}{|c|}{ Method } & Linear & Polynomial & RBF \\
\hline LDP & $92.8 \pm 1.7$ & $92.8 \pm 1.7$ & $94.3 \pm 3.9$ \\
\hline LDNP & $92.3 \pm 3.0$ & $95.1 \pm 4.1$ & $94.8 \pm 3.1$ \\
\hline DR-LDP & $91.5 \pm 1.7$ & $91.9 \pm 1.3$ & $93.4 \pm 2.9$ \\
\hline MDR-LDP & $92.8 \pm 2.5$ & $95.4 \pm 2.5$ & $96.3 \pm 2.1$ \\
\hline RC-LDP & $94.2 \pm 1.2$ & $98.3 \pm 1.5$ & $98.5 \pm 0.8$ \\
\hline
\end{tabular}


Table 6: Confusion matrix of six-class expression recognition rate of RC-LDP on CK database

\begin{tabular}{|l|l|l|l|l|l|l|}
\hline RC-LDP & Anger & Disgust & Fear & Happy & Sad & Surprise \\
\hline Anger & 99.12 & 0 & 0.17 & 0 & 0.35 & 0.36 \\
\hline Disgust & 0 & 100.00 & 0 & 0 & 0 & 0 \\
\hline Fear & 0.20 & 0 & 99.70 & 0 & 0.10 & 0 \\
\hline Happy & 0 & 0 & 0 & 99.70 & 0.20 & 0.10 \\
\hline Sad & 0.20 & 0 & 0.30 & 0 & 99.50 & 0 \\
\hline Surprise & 0 & 0 & 0 & 0.20 & 0.10 & 99.70 \\
\hline
\end{tabular}

Table 7: Confusion matrix of seven-class expression recognition rate of RC-LDP on CK database

\begin{tabular}{|l|l|l|l|l|l|l|l|}
\hline RC-LDP & Anger & Disgust & Fear & Happy & Sad & Surprise & Neutral \\
\hline Anger & 93.56 & 0 & 0.85 & 0 & 0 & 3.46 & 2.13 \\
\hline Disgust & 0.50 & 97.25 & 0 & 0 & 0 & 0 & 2.25 \\
\hline Fear & 0.14 & 0 & 98.99 & 0 & 0.20 & 0 & 0.67 \\
\hline Happy & 0 & 0 & 0 & 98.25 & 0 & 0 & 1.75 \\
\hline Sad & 0.18 & 0.09 & 0 & 0 & 98.83 & 0 & 0.90 \\
\hline Surprise & 0 & 0 & 0 & 0.25 & 0 & 98.75 & 1.00 \\
\hline Neutral & 1.25 & 0 & 1.37 & 0 & 0.84 & 0 & 96.54 \\
\hline
\end{tabular}

\subsection{JAFFE Database}

The Japanese Female Facial Expression (JAFFE) database [3] consists of 213 images of females using 10 subjects. Each image has a size of $256 \times 256$. Each category of expression has almost the same number of images. The database consists of frontal pose image and their hair was tied back to expose all the expressive areas of the face without occlusion.

The performance on the JAFFE database is evaluated with six-class and seven-class expression. The recognition rate of different class expressions using the SVM classifier is evaluated to show the efficiency of the proposed method in facial expression recognition. Tables 8 and 9 demonstrates the recognition rate of the proposed method with other state-of-the-art methods. The proposed method attained better recognition rate in six-class and seven-class expression dataset compared with other local descriptors. The confusion matrix of six-class and seven-class expression of the proposed method presented in Tables 10 and 11.

Table 8: Recognition rate of six-class expression of RC-LDP using SVM on JAFFE database

\begin{tabular}{|l|c|l|c|}
\hline \multicolumn{1}{|c|}{ Method } & Linear & Polynomial & RBF \\
\hline LDP & $89.9 \pm 5.2$ & $89.9 \pm 5.2$ & $90.1 \pm 4.9$ \\
\hline LDNP & $92.9 \pm 1.7$ & $93.4 \pm 2.2$ & $92.3 \pm 1.7$ \\
\hline DR-LDP & $91.5 \pm 2.1$ & $92.4 \pm 1.5$ & $93.3 \pm 1.2$ \\
\hline MDR-LDP & $93.3 \pm 1.3$ & $94.6 \pm 1.7$ & $95.4 \pm 1.5$ \\
\hline RC-LDP & $95.2 \pm 3.2$ & $95.2 \pm 3.7$ & $96.3 \pm 2.8$ \\
\hline
\end{tabular}

\subsection{EMOTIW 2015 Database}

The EmotiW 2015 benchmark [24] includes data from version 2.0 of the Static Facial Expression in the Wild benchmark (SFEW). The images are selected from different videos of the Acted Facial Expressions in the Wild (AFEW). In EmotiW 2015 database, the images captured under an unconstrained environment. It consists of a wide range of head poses and ages, both genders, different occlusions and resolution qualities [25]. Table 
Table 9: Recognition rate of seven-class expression of RC-LDP using SVM on JAFFE database

\begin{tabular}{|l|c|l|c|}
\hline \multicolumn{1}{|c|}{ Method } & Linear & Polynomial & RBF \\
\hline LDP & $84.9 \pm 4.7$ & $84.9 \pm 4.7$ & $85.4 \pm 4.0$ \\
\hline LDNP & $90.1 \pm 3.0$ & $91.1 \pm 4.1$ & $89.2 \pm 3.1$ \\
\hline DR-LDP & $89.5 \pm 3.7$ & $90.7 \pm 3.5$ & $90.1 \pm 3.9$ \\
\hline MDR-LDP & $91.6 \pm 2.5$ & $92.1 \pm 3.0$ & $92.7 \pm 2.1$ \\
\hline RC-LDP & $92.3 \pm 1.8$ & $93.4 \pm 3.6$ & $93.8 \pm 1.9$ \\
\hline
\end{tabular}

Table 10: Confusion matrix of six-class expression recognition rate of RC-LDP on JAFFE database

\begin{tabular}{|l|l|l|l|l|l|l|}
\hline RC-LDP & Anger & Disgust & Fear & Happy & Sad & Surprise \\
\hline Anger & 96.88 & 0.80 & 1.20 & 0 & 1.12 & 0 \\
\hline Disgust & 1.80 & 95.23 & 0.70 & 0 & 2.27 & 0 \\
\hline Fear & 0.60 & 0 & 96.46 & 0 & 1.15 & 1.79 \\
\hline Happy & 0 & 0 & 0.82 & 98.33 & 0 & 0.85 \\
\hline Sad & 0.93 & 1.65 & 1.11 & 0 & 95.61 & 0.70 \\
\hline Surprise & 0 & 0 & 0.47 & 1.89 & 0.36 & 97.28 \\
\hline
\end{tabular}

Table 11: Confusion matrix of seven-class expression recognition rate of RC-LDP on JAFFE database

\begin{tabular}{|l|l|l|l|l|l|l|l|}
\hline RC-LDP & Anger & Disgust & Fear & Happy & Sad & Surprise & Neutral \\
\hline Anger & 90.21 & 2.94 & 2.05 & 0 & 0 & 0 & 4.80 \\
\hline Disgust & 3.87 & 88.92 & 0 & 0 & 2.89 & 0 & 4.32 \\
\hline Fear & 1.43 & 1.25 & 91.76 & 0 & 1.29 & 2.64 & 1.63 \\
\hline Happy & 0 & 0 & 0 & 98.03 & 0 & 0.80 & 1.17 \\
\hline Sad & 3.26 & 3.56 & 4.23 & 0 & 85.98 & 0 & 2.97 \\
\hline Surprise & 0 & 0 & 0 & 1.87 & 0 & 94.60 & 3.53 \\
\hline Neutral & 1.42 & 0 & 1.25 & 0.84 & 1.06 & 0 & 95.43 \\
\hline
\end{tabular}

12 shows the confusion matrix of the seven-class expression recognition rate on SFEW. Due to variations of poses and occlusion, the results are not satisfactory. Particularly disgust and fear attained inferior performance because the expressions are confused with anger, happiness, sadness and surprise.

Table 12: Confusion matrix of seven-class expression recognition rate of RC-LDP on SFEW 2.0

\begin{tabular}{|l|l|l|l|l|l|l|l|}
\hline RC-LDP & Anger & Disgust & Fear & Happy & Sad & Surprise & Neutral \\
\hline Anger & 67.89 & 1.65 & 3.88 & 8.98 & 0.80 & 7.34 & 9.46 \\
\hline Disgust & 18.75 & 16.80 & 6.32 & 18.56 & 4.27 & 18.95 & 16.35 \\
\hline Fear & 16.75 & 2.25 & 15.69 & 10.42 & 19.86 & 20.47 & 14.56 \\
\hline Happy & 0 & 0.60 & 0.49 & 87.45 & 3.93 & 2.76 & 4.77 \\
\hline Sad & 8.12 & 0 & 5.43 & 8.67 & 42.56 & 22.66 & 12.56 \\
\hline Surprise & 0.75 & 0.48 & 1.93 & 6.73 & 8.45 & 80.34 & 1.32 \\
\hline Neutral & 4.56 & 0.98 & 1.56 & 1.17 & 7.41 & 20.05 & 64.27 \\
\hline
\end{tabular}




\section{Conclusion}

In this paper, an RC-LDP local descriptor proposed for facial analysis. The novel descriptor combined with two novel descriptors, namely row based local directional pattern and correlation based local directional pattern. RC-LDP computes informative features to represent the face image. This hybrid method helps to detect the face in all the major challenges highlighted above. It provides better recognition rate in the face and facial expression recognition. The face recognition tested on FERET, Extended YALE-B, ORL, LFW for verification and AR database. For facial expression, the experimentation carried out on CK, JAFEE and EmotiW 2015 databases. The experimental results show that the proposed method attained better recognition rate than other local descriptors.

\section{References}

[1] Ojala, Timo and Pietikäinen, Matti and Harwood, David, "A comparative study of texture measures with classification based on featured distributions", Pattern recognition, Vol. 26, No. 1, pp. 51-59, 1996.

[2] Sirovich, Lawrence and Kirby, Michael, "The AR Face Database”, CVC Technical Report, 1998.

[3] Lyons, Michael and Akamatsu, Shigeru and Kamachi, Miyuki and Gyoba, Jiro, "Coding facial expressions with gabor wavelets", IEEE International Conference on Automatic Face and Gesture Recognition, pp 200-205, 1998.

[4] Kanade, Takeo and Cohn, Jeffrey F and Tian, Yingli, "Comprehensive database for facial expression analysis",International Conference on Automatic Face and Gesture Recognition, pp. 46-53, 2000.

[5] Phillips, P Jonathon and Moon, Hyeonjoon and Rizvi, Syed A and Rauss, Patrick J, "The FERET evaluation methodology for face-recognition algorithms",IEEE Transactions on Pattern Analysis and Machine Intelligence, Vol 22, No 10, pp.1090-1104, 2000.

[6] AT and TLaboratories, "Database of Faces", URL : http://www.cl.cam.ac.uk/research/dtg/attarchivelfacedatabase.html, 2002.

[7] Ojala, Timo and Pietikäinen, Matti and Mäenpää, Topi,"Multiresolution gray-scale and rotation invariant texture classification with local binary patterns",IEEE Transactions on Pattern Analysis and Machine Intelligence, Vol 24, No 7, pp. 971-987, 2002.

[8] Ahonen, Timo and Hadid, Abdenour and Pietikäinen, Matti, "Face recognition with local binary patterns", International Jounal of Computer Vision, pp. 469-481, 2004.

[9] Lee, Kuang-Chih and Ho, Jeffrey and Kriegman, David J, "Acquiring linear subspaces for face recognition under variable lighting", IEEE Transactions on Pattern Analysis and Machine Intelligence, Vol 27, No 5, pp.684-698, 2005.

[10] Ahonen, Timo and Hadid, Abdenour and Pietikainen, Matti, "Face description with local binary patterns: Application to face recognition", IEEE Transactions on Pattern Analysis and Machine Intelligence, Vol.28 , No. 12 ,pp. 2037-2041, 2006.

[11] Huang, Gary B and Ramesh, Manu and Berg, Tamara and Learned-Miller, Erik, "Labeled faces in the wild: A database for studying face recognition in unconstrained environments", Technical Report 07-49, University of Massachusetts, Amherst, 2007.

[12] Hadid, Abdenour and Zhao, Guoying and Ahonen, Timo and Pietikäinen, Matti, "Face analysis using local binary patterns", Handbook of Texture Analysis, pp. 347-373, 2008. 
[13] Shan, Caifeng and Gong, Shaogang and McOwan, Peter W, "Facial expression recognition based on local binary patterns: A comprehensive study", Image and Vision Computing, Vol.27, No.6, pp. 803-816, 2009.

[14] Jabid, Taskeed and Kabir, Md Hasanul and Chae, Oksam, "Local directional pattern (ldp) for face recognition”, International Conference on Consumer Electronics, pp. 329-330, 2010.

[15] Jabid, Taskeed and Kabir, Md Hasanul and Chae, Oksam, "Local directional pattern (LDP)-A robust image descriptor for object recognition",International Conference on Advanced Video and Signal Based Surveillance, pp. 482-487, 2010.

[16] Jabid, Taskeed and Kabir, Md Hasanul and Chae, Oksam, "Facial expression recognition using local directional pattern (LDP)",International Conference on Image Processing, pp. 1605-1608, 2010.

[17] Tan, Xiaoyang and Triggs, Bill, "Enhanced local texture feature sets for face recognition under difficult lighting conditions”, ETRI journal, Vol. 19, No.6, pp. 1635-1650, 2010.

[18] Zhang, Baochang and Gao, Yongsheng and Zhao, Sanqiang and Liu, Jianzhuang, "Local derivative pattern versus local binary pattern: face recognition with high-order local pattern descriptor", IEEE Transactions on Image Processing, Vol. 19, No. 2, pp. 533-544, 2010.

[19] Wolf, Lior and Hassner, Tal and Taigman, Yaniv, "Effective unconstrained face recognition by combining multiple descriptors and learned background statistics", IEEE Transactions on pattern analysis and machine intelligence, Vol. 33, No. 10, pp. 1978-1990.

[20] Hasanul Kabir, Taskeed Jabid and Chae, Oksam, "Local Directional Pattern Variance (LDPv): A robust feature descriptor for facial expression recognition", Int Arab J Inf Technol, Vol. 9, No. 4, pp. 382-391, 2012.

[21] Ishraque, SM Zahid and Banna, AKM Hasanul and Chae, Oksam (2012), "Local Gabor directional pattern for facial expression recognition", International Conference on Computer and Information Technology, pp. 164-167, 2012.

[22] Ramirez Rivera, Adin and Rojas Castillo, Jorge and Chae, Oksam (2013), "Local directional number pattern for face analysis: Face and expression recognition", IEEE Transactions on Image Processing, Vol 22, No 5, pp. 1740-1752, 2013.

[23] Zhong, Fujin and Zhang, Jiashu, "Face recognition with enhanced local directional patterns", Neurocomputing, Vol 119, No 5, pp. 375-384, 2013.

[24] Dhall, Abhinav and Ramana Murthy, OV and Goecke, Roland and Joshi, Jyoti and Gedeon, Tom, "Video and image based emotion recognition challenges in the wild: Emotiw 2015", International Conference on Multimodal Interaction, pp.423-426, 2015.

[25] Levi, Gil and Hassner, Tal, "Emotion recognition in the wild via convolutional neural networks and mapped binary patterns", International Conference on Multimodal Interaction, pp.503-510, 2015.

[26] Liu, Jun and Jing, Xiaojun and Sun, Songlin and Lian, Zifeng , "Local Gabor dominant direction pattern for face recognition", Chinese Journal of Electronics, Vol 24, No 2, pp. 245-250, 2015.

[27] Nguyen, Huu-Tuan and Caplier, Alice, "Local patterns of gradients for face recognition", IEEE Transactions on Information Forensics and Security, Vol 10, No 8 , pp. 1739-1751, 2015.

[28] Fathi, Abdolhossein and Alirezazadeh, Pendar and Abdali-Mohammadi, Fardin, "A new Global-GaborZernike feature descriptor and its application to face recognition", Journal of Visual Communication and Image Representation, Vol 38, pp. 65-72, 2016. 
[29] Srinivasa Perumal.R and Chandra Mouli P.V.S.S.R, "A Comparative Analysis of Local Pattern Descriptors for Face Recognition ”, Knowledge Computing and its Applications, Springer, pp. 129-154, 2018.

[30] Srinivasa Perumal, R and Chandra Mouli, P.V.S.S.R , "Two-level dimensionality reduced local directional pattern for face recognition”, International Journal of Biometrics, Vol 8, No 1, pp. 52-64, 2016.

[31] Srinivasa Perumal, R and Chandra Mouli, P.V.S.S.R, "Dimensionality reduced local directional pattern (DR-LDP) for face recognition”, Expert Systems with Applications, Vol 63, pp.66-73, 2016.

[32] Yoo, Cheol-Hwan and Kim, Seung-Wook and Jung, June-Young and Ko, Sung-Jea (2017), "Highdimensional feature extraction using bit-plane decomposition of local binary patterns for robust face recognition", Journal of Visual Communication and Image Representation, Vol 45, pp.11-19, 2017.

[33] Srinivasa Perumal, R and Chandra Mouli, P.V.S.S.R ,"Dimensionality reduced local directional number pattern for face recognition", Journal of Ambient Intelligence and Humanized Computing, Vol 9, No 1, pp. 95-103, 2018.

[34] Srinivasa Perumal, R and Chandra Mouli, P.V.S.S.R , "Modified dimensionality reduced local directional pattern for facial analysis", Journal of Ambient Intelligence and Humanized Computing, Vol 9, No 3 , pp. 725-737, 2018. 